\title{
Diez portainjertos de vid: Efectos sobre el crecimiento, la producción y la composición de la uva del cv. Sauvignon blanc en la denominación de origen rueda (España) / Ten grapevine rootstocks: Effects on vegetative growth, production and grape composition of cv. Sauvignon Blanc in the Appellation of Origin Rueda (Spain)
}

\author{
Jesus Yuste $^{1}$, Alejandro Vicente ${ }^{2}$, Enrique Barajas $^{1}$, y Maria Alburquerque ${ }^{2}$ \\ ${ }^{1}$ Instituto Tecnologico Agrario de Castilla y Leon, 47071 Valladolid, Spain \\ ${ }^{2}$ At present: external viticulture activity, Spain
}

\begin{abstract}
The knowledge of the behaviour of different grapevine rootstocks is basic to achieve a good adaptation of vine to its growing area. With the objective of knowing the agronomic and qualitative response of cv. Sauvignon Blanc to the use of several rootstocks, a trial was established in 2006 in the D.O. Rueda. The vines are vertical trellised, with bilateral Royat cordon pruning, and the vine spacing is $2.60 \mathrm{~m} \times 1.25 \mathrm{~m}$. The row orientation is NNW $\left(\mathrm{N}-25^{\circ}\right)$. The experimental design consisted of randomized blocks with 4 replications and elemental plot of 60 vines. Over the period 2010-2014 it has been developed the study of the following 10 rootstocks (treatments): 110R, 101-14M, 420A, 3309C, 41B, 161-49C, 196-17C, Fercal, Gravesac and RGM. The rootstocks $420 \mathrm{~A}$ and $41 \mathrm{~B}$ showed a production higher than $50 \%$ with respect to 196-17C and 161-49C, and higher to $100 \%$ than RGM, due to the number of clusters per vine and, in greater amount, to the cluster weight. Fercal and Gravesac showed an increase of pruning wood weight of $24 \%$ with respect to $196-17 \mathrm{C}$ and $161-49 \mathrm{C}$, and $90 \%$ with respect to RGM, mainly due to the shoot weight. The sugar concentration increased with 101-14M, 196-17C and Fercal, and became reduced with 161-49C, 41B and RGM. The $\mathrm{pH}$ of must was reduced with Fercal whereas the titratable acidity increased, which also showed increase with Gravesac and 161-49C. The tartaric acid hardly increased slightly with Fercal and 161-49C, whereas the malic acid increased with Gravesac and Fercal, and was reduced with 41B, 3309C, RGM and 101-14M. The potassium concentration increased with 196-17C, Gravesac and Fercal, and was reduced with 41B, 161-49C, 420A and 3309C. The effects observed show alternatives for rootstock election according to the growing conditions and objectives of the vineyard.
\end{abstract}

\section{Introducción}

La utilización del portainjerto en el cultivo de la vid tuvo su origen en la lucha contra la filoxera. Sin embargo, actualmente los portainjertos se emplean también para mejorar la adaptación de la vid al terreno, como por ejemplo en los suelos calizos, y para controlar ciertas plagas del suelo, además de la filoxera, como los nemátodos [1].

Los primeros trabajos de mejora de portainjertos en el mundo estuvieron orientados a conseguir resistencia a la caliza y a la filoxera, pero progresivamente se han ido obteniendo portainjertos que presentan además otras cualidades. Dichas cualidades suponen criterios añadidos a considerar en la elección de portainjerto, como son el vigor que éste confiere a la variedad, la influencia sobre el ciclo vegetativo del injerto, la facilidad de estaquillado y de injerto, la adaptación a condiciones específicas del lugar (sequía, humedad, salinidad, etc.) y la calidad de la uva [2].

El uso de distintos portainjertos conlleva respuestas variables, de las diferentes variedades de vid, en las diversas regiones y zonas productoras de vino del mundo, las cuales dependen de las condiciones edafoclimáticas del lugar de cultivo [3]. Así, la elección adecuada del portainjerto, en función de las características del lugar de cultivo y los objetivos productivos, puede permitir el incremento de la eficiencia productiva del viñedo, y por consiguiente el aumento de la rentabilidad del viticultor [4]. El análisis de los factores de producción y los criterios para la elección de portainjerto deben ser considerados como aspectos preferentes en términos tanto de experimentación como de aplicación práctica en la plantación de viñedo. En definitiva, se trata de un factor de producción que lleva implícita una enorme repercusión en la rentabilidad del viñedo, en el mismo sentido que ocurre, por ejemplo, con la mejora de la eficiencia hídrica y fisiológica del viñedo [5], las técnicas de riego subterráneo, el sistema RDI (regulated deficit irrigation) o el PRD (partial root-zone drying), la mecanización integral u otras operaciones vitivinícolas alternativas. Muchas de las citadas técnicas suelen estar encaminadas a paliar el déficit hídrico severo o el exceso de vigor y diversos inconvenientes de conducción del viñedo. En este ámbito, el portainjerto puede proporcionar respuestas permanentes 
que ayuden a resolver este tipo de problemas, sobre todo los relacionados con la escasez de agua o con el control del desarrollo vegetativo del viñedo [6].

En España, a pesar de ser el país con mayor superficie de cultivo de viñedo para vinificación del mundo, cercana a un millón de hectáreas, se utiliza mayoritariamente un número reducido de portainjertos, la mayoría de los cuales pertenece a especies de origen americano del género Vitis, concretamente a cruzamientos entre dichas especies, $V$. riparia, $V$. rupestris y $V$. berlandieri, así como a su cruzamiento con $V$. vinifera. De entre ellos destacan los portainjertos 110 Richter, sobre todo, y 41B, empleado específicamente en zonas con suelos calizos [7]. Sin embargo, la existencia de zonas productoras con diferentes características edafoclimáticas sugiere la conveniencia de conocer el funcionamiento de portainjertos alternativos que puedan mejorar la adaptación del viñedo dependiendo de las condiciones de la zona de cultivo [8]. Esta situación se contempla también en la región de Castilla y León, donde ya se han llevado a cabo algunos trabajos con portainjertos, entre los que cabe mencionar los realizados con Tempranillo en la D.O. Rueda [7], en la D.O. Toro $[6,8]$ y en la D.O. Cigales [9], con Sauvignon Blanc en la D.O. Rueda [10], con Verdejo en la D.O. Rueda [11] y con Mencía en la D.O. Bierzo [12].

Por tanto, se considera conveniente profundizar en el estudio de portainjertos, entre los cuales habría que incluir, además de los citados 110R y 41B, otros de diferentes características, como 140Ru, 1103P, 101-14M, 420A, 5BB, 161-49C, 333EM y SO4, para conocer su influencia en las diversas zonas de Castilla y León.

Con el ánimo de conocer los efectos del portainjerto en el comportamiento agronómico del viñedo, se ha planteado un ensayo experimental con un grupo de 10 portainjertos de vid, encaminado a evaluar la influencia de los mismos en el cv. Sauvignon Blanc, a través de la cuantificación de parámetros vegetativos, productivos y de calidad de la uva, en la Denominación de Origen Rueda, ubicada en el centro de Castilla y León.

\section{Material y métodos}

El estudio del ensayo se ha realizado, a lo largo del periodo 2010-2014, en la localidad de Medina del Campo (Valladolid, España), en un viñedo perteneciente a las bodegas Grupo Yllera S.L., ubicado dentro de la D.O. Rueda, en el centro de Castilla y León. Las coordenadas del ensayo son $41^{\circ} 21^{\prime} 02^{\prime \prime} \mathrm{N} \mathrm{y} 4^{\circ} 56^{\prime} 15^{\prime \prime} \mathrm{O}$.

Las cepas del viñedo experimental, plantadas en 2006, son del cv. Sauvignon Blanc y están injertadas sobre un grupo de distintos portainjertos, que constituyen los tratamientos experimentales objeto de estudio, que son: 110R, 101-14M, 420A, 3309C, 41B, 161-49C, 196-17C, Fercal, Gravesac y Riparia Gloire de Montpellier (RGM).

Las cepas han sido conducidas en espaldera, con formación en cordón Royat bilateral y posicionamiento vertical de la vegetación (VSP). La orientación de filas es $\mathrm{NNO}\left(\mathrm{N}-25^{\circ}\right)$. El marco de plantación es de 2,60 $\mathrm{m} \times$ $1,25 \mathrm{~m}$, que corresponde a un área de suelo de $3,25 \mathrm{~m}^{2} /$ cepa y a una densidad de 3.077 cepas/ha. La carga de poda ha sido de 16 yemas por cepa, en 8 pulgares de 2 yemas. Se ha aplicado anualmente una operación de poda en verde, tras el período de riesgo de helada primaveral, para el ajuste de la carga a 16 pámpanos por cepa.
El diseño experimental es en bloques al azar, con 4 repeticiones de 60 cepas y parcela elemental de 20 cepas de control.

El suelo del ensayo es suficientemente profundo para el desarrollo del viñedo, con un horizonte superior de textura arcillosa, y presenta una suave pendiente homogénea y decreciente en el sentido este-oeste. El régimen hídrico de cultivo durante el estudio se ha mantenido a través de riego deficitario, con una aplicación de agua del $25 \%$ de ETo, desde parada de crecimiento vegetativo de pámpanos principales hasta vendimia.

El análisis estadístico de los resultados se ha realizado mediante análisis de varianza (ANOVA), con el programa STATISTICA 7.0.

Los datos medios mensuales de pluviometría y temperatura, correspondientes al periodo 2010-2014, se detallan en la tabla siguiente.

Tabla 1. Datos termo-pluviométricos medios del periodo 2010-2014 (octubre-2009 / septiembre-2014), registrados en Rueda (Valladolid). Tm: temperatura media $\left({ }^{\circ} \mathrm{C}\right)$, Tmax: temperatura media de máximas $\left({ }^{\circ} \mathrm{C}\right)$, Tmin: temperatura media de mínimas $\left({ }^{\circ} \mathrm{C}\right)$, P: precipitación $(\mathrm{mm})$.

\begin{tabular}{ccccc}
\hline & Tm & Tmax & Tmin & P \\
\hline Oct & 13,0 & 20,3 & 6,5 & 37,1 \\
Nov & 7,6 & 12,4 & 3,3 & 19,3 \\
\hline Dic & 3,9 & 8,4 & $-0,2$ & 41,3 \\
Ene & 4,2 & 8,4 & 0,2 & 32,4 \\
\hline Feb & 4,2 & 9,8 & $-1,1$ & 25,7 \\
Mar & 8,1 & 14,5 & 2,0 & 44,6 \\
\hline Abr & 11,1 & 17,1 & 4,9 & 35,6 \\
May & 14,8 & 21,7 & 7,6 & 17,4 \\
\hline Jun & 19,1 & 26,7 & 10,9 & 14,8 \\
Jul & 22,1 & 30,3 & 13,3 & 6,4 \\
\hline Ago & 21,9 & 30,2 & 13,4 & 2,5 \\
Sep & 18,2 & 26,0 & 10,9 & 28,0 \\
\hline Año & 12,3 & 18,8 & 6,0 & 305 \\
\hline
\end{tabular}

\section{Resultados y discusión}

\subsection{Producción de uva}

El rendimiento en uva ha mostrado la influencia del empleo de los diferentes portainjertos, con diferencias estadísticamente significativas observadas a favor fundamentalmente de $420 \mathrm{~A}$ y $41 \mathrm{~B}$, que han propiciado una mayor producción media de uva, en torno a $2,2 \mathrm{~kg} / \mathrm{cepa}$, con proximidad de los portainjertos 110R y 3309C, en torno a 1,9 kg/cepa (Tabla 2). Los tratamientos RGM, con menos de $1 \mathrm{~kg} /$ cepa, y 196-17C y $161-49 \mathrm{C}$, con 1,4 $\mathrm{kg} / \mathrm{cepa}$, han mostrado un rendimiento claramente inferior al resto. Los portainjertos 420A y 41B han mostrado un incremento medio de rendimiento superior al $50 \%$ respecto a los tratamientos 196-17C y 161-49C.

El número de racimos por cepa ha estado estrechamente relacionado con la producción de uva, destacando significativamente $420 \mathrm{~A}$ y $41 \mathrm{~B}$, con promedio de 21,6 racimos, frente a 196-17C y 161-49C, con 17,9 racimos, $\mathrm{y}$, sobre todo, RGM, con 15,0 racimos. El incremento de 17,9 a 21,6 racimos supone un $20 \%$. Las diferencias de rendimiento han sido debidas en mayor 
Tabla 2. Número de racimos, N.rac.; Producción de uva, Prod. (kg/cepa); Peso de racimo, P.rac. (g); Peso de baya, P.bay. (g); Número de bayas del racimo, N.bay.; de los 10 portainjertos (PI). Promedio del periodo 2010-2014. Significación estadística $*=\mathrm{p}<5 \%$; letras distintas indican valores estadísticos significativamente diferentes (para todas las tablas).

\begin{tabular}{cccccc}
\hline PI & N.rac. & Prod. & P.rac. & P.bay. & N.bay. \\
\hline 110R & $21,2^{\mathrm{ab}}$ & $1,95^{\mathrm{ab}}$ & $91,7^{\mathrm{ab}}$ & $1,22^{\mathrm{ab}}$ & $76,7^{\mathrm{ab}}$ \\
101-14M & $19,2^{\mathrm{bc}}$ & $1,63^{\mathrm{bc}}$ & $84,7^{\mathrm{bc}}$ & $1,11^{\mathrm{b}}$ & $78,3^{\mathrm{ab}}$ \\
420A & $21,7^{\mathrm{a}}$ & $2,29^{\mathrm{a}}$ & $104,9^{\mathrm{a}}$ & $1,27^{\mathrm{a}}$ & $83,9^{\mathrm{a}}$ \\
3309C & $20,9^{\mathrm{ab}}$ & $1,91^{\mathrm{ab}}$ & $91,3^{\mathrm{ab}}$ & $1,21^{\mathrm{ab}}$ & $78,4^{\mathrm{ab}}$ \\
41B & $21,5^{\mathrm{a}}$ & $2,11^{\mathrm{a}}$ & $97,5^{\mathrm{ab}}$ & $1,20^{\mathrm{ab}}$ & $82,4^{\mathrm{a}}$ \\
$\mathbf{1 6 1 - 4 9 C}$ & $18,1^{\mathrm{c}}$ & $1,42^{\mathrm{c}}$ & $78,6^{\mathrm{c}}$ & $1,21^{\mathrm{ab}}$ & $66,9^{\mathrm{bc}}$ \\
196-17C & $17,7^{\mathrm{c}}$ & $1,41^{\mathrm{c}}$ & $78,8^{\mathrm{c}}$ & $1,16^{\mathrm{ab}}$ & $68,9^{\mathrm{bc}}$ \\
Fercal & $20,3^{\mathrm{ab}}$ & $1,81^{\mathrm{b}}$ & $89,0^{\mathrm{bc}}$ & $1,18^{\mathrm{ab}}$ & $76,7^{\mathrm{ab}}$ \\
Gravesac & $18,8^{\mathrm{bc}}$ & $1,59^{\mathrm{bc}}$ & $83,7^{\mathrm{bc}}$ & $1,19^{\mathrm{ab}}$ & $71,2^{\mathrm{b}}$ \\
RGM & $15,0^{\mathrm{d}}$ & $0,93^{\mathrm{d}}$ & $62,8^{\mathrm{d}}$ & $1,07^{\mathrm{b}}$ & $59,7^{\mathrm{c}}$ \\
Sig. & $*$ & $*$ & $*$ & $*$ & $*$ \\
\hline
\end{tabular}

Tabla 3. Número de sarmientos francos, Franc.; Número de chupones, Chup.; y Número total de sarmientos, S.tot., por cepa; Peso de madera de poda, PMP (g/cepa); Peso de sarmiento, P.sar. (g); Indice de Ravaz, I.Rz.; de los 10 portainjertos (PI). Promedio del periodo 2010-2014.

\begin{tabular}{ccccccc}
\hline PI & Franc. & Chup. & S.tot. & PMP & P.sar. & I.Rz. \\
\hline 110R & 14,69 & $1,67^{\mathrm{b}}$ & 16,0 & $970^{\mathrm{ab}}$ & $63,0^{\mathrm{ab}}$ & $2,01^{\mathrm{ab}}$ \\
$\mathbf{1 0 1 - 1 4 M}$ & 14,35 & $1,93^{\mathrm{ab}}$ & 15,9 & $932^{\mathrm{b}}$ & $58,9^{\mathrm{b}}$ & $1,76^{\mathrm{b}}$ \\
\hline $\mathbf{4 2 0 A}$ & 14,69 & $1,92^{\mathrm{ab}}$ & 16,3 & $993^{\mathrm{ab}}$ & $61,5^{\mathrm{ab}}$ & $2,31^{\mathrm{a}}$ \\
3309C & 14,72 & $1,68^{\mathrm{b}}$ & 16,1 & $885^{\mathrm{bc}}$ & $55,5^{\mathrm{c}}$ & $2,17^{\mathrm{a}}$ \\
41B & 14,75 & $2,13^{\mathrm{a}}$ & 16,5 & $924^{\mathrm{b}}$ & $56,6^{\mathrm{bc}}$ & $2,30^{\mathrm{a}}$ \\
161-49C & 14,81 & $1,13^{\mathrm{c}}$ & 15,6 & $846^{\mathrm{c}}$ & $54,1^{\mathrm{c}}$ & $1,68^{\mathrm{b}}$ \\
196-17C & 14,63 & $1,90^{\mathrm{ab}}$ & 16,2 & $820^{\mathrm{c}}$ & $51,4^{\mathrm{c}}$ & $1,72^{\mathrm{b}}$ \\
Fercal & 14,94 & $1,62^{\mathrm{b}}$ & 16,1 & $1049^{\mathrm{a}}$ & $65,3^{\mathrm{a}}$ & $1,72^{\mathrm{b}}$ \\
Gravesac & 14,17 & $1,59^{\mathrm{b}}$ & 15,4 & $1017^{\mathrm{a}}$ & $66,8^{\mathrm{a}}$ & $1,57^{\mathrm{b}}$ \\
RGM & 14,77 & $1,16^{\mathrm{c}}$ & 15,5 & $541^{\mathrm{d}}$ & $34,9^{\mathrm{d}}$ & $1,75^{\mathrm{b}}$ \\
Sig. & - & $*$ & - & $*$ & $*$ & $*$ \\
\hline
\end{tabular}

medida a la variación del peso del racimo, que en los dos portainjertos más productivos ha mostrado un promedio de $101 \mathrm{~g}$ y en los menos productivos citados ha sido de $79 \mathrm{~g}$; o sea, en torno a un $30 \%$ mayor en los primeros, lo que ha supuesto que las diferencias entre ambos grupos fuesen estadísticamente significativas. Evidentemente, el portainjerto menos productivo, RGM, mostró asimismo un peso de racimo significativamente muy inferior, de solo $63 \mathrm{~g}$.

El peso de baya también ha variado entre los distintos tratamientos, aunque las diferencias estadísticamente significativas han sido menores. El portainjerto 420A ha mostrado una baya de tamaño significativamente mayor, con $1,27 \mathrm{~g}$, que RGM y 101-14, con 1,07 y $1,11 \mathrm{~g}$ respectivamente. Las diferencias en peso de baya entre los tratamientos restantes no resultaron estadísticamente significativas. El número de bayas del racimo fue significativamente superior en los dos portainjertos más productivos, 420A y 41B, con 83 bayas, que en 161-49C y 196-17C, con 68 bayas, y sobre todo que en RGM, con apenas 60 bayas por racimo. Por tanto, el número de bayas del racimo ha contribuido generalmente en mayor medida que el peso de baya a las diferencias en el peso del racimo entre tratamientos.

En resumen, los portainjertos utilizados han provocado claras diferencias en el rendimiento en uva, destacando
420A y 41B como más productivos, y RGM, 196-17C y 161-49C como menos productivos, en relación más estrecha con el tamaño del racimo que con el número de racimos.

\subsection{Desarrollo vegetativo}

El número total de sarmientos por cepa ha sido muy similar en todos los tratamientos, oscilando entre 15,4 y 16,5, gracias al ajuste de carga de brotes que se aplicó en las semanas posteriores a la fecha de brotación (Tabla 3). Las escasas diferencias observadas entre algunos portainjertos, que no han sido estadísticamente significativas, se debieron a las diferencias en el desarrollo de chupones, que resultaron significativamente favorables a 41B y desfavorables a 161-49C y RGM, puesto que el número de sarmientos francos resultó muy similar en todos los tratamientos.

El peso de madera de poda fue significativamente superior en los tratamientos Fercal y Gravesac, con más de $1 \mathrm{~kg} /$ cepa, que en 196-17C y 161-49C, con menos de $850 \mathrm{~g} /$ cepa, y sobre todo que en RGM, con $540 \mathrm{~g} /$ cepa. Los portainjertos Fercal y Gravesac han mostrado un incremento medio del peso de madera de poda del $24 \%$ con respecto a los tratamientos 196-17C y 161-49C, y del $90 \%$ con respecto a RGM.

La tendencia del vigor del sarmiento ha sido muy similar a la del peso de madera de poda, destacando nuevamente Gravesac y Fercal, con 66 g/sarmiento, frente a 196-17C, 161-49C y 3309C, con valores en torno a $53 \mathrm{~g} /$ sarmiento, y sobre todo frente a RGM, con un sarmiento de $35 \mathrm{~g}$, muy significativamente inferior.

El índice de Ravaz también ha mostrado diferencias significativas entre tratamientos, favorables a los portainjertos más productivos, 420A y 41B, y a 3309C, con respecto al resto de tratamientos, entre los cuales no se han observado diferencias estadísticamente significativas. Los bajos valores de este índice observados indican que se trata de unas condiciones de cultivo del viñedo con un nivel productivo bastante moderado.

Atendiendo a los resultados obtenidos, los portainjertos Fercal y Gravesac han mostrado un mayor desarrollo vegetativo, mientras 196-17C, 161-49C y, sobre todo, RGM han mostrado un desarrollo claramente inferior al resto de portainjertos.

\subsection{Composición de la uva}

La concentración de azúcares se ha visto ligeramente afectada por los portainjertos, con diferencias de escasa magnitud (Tabla 4). Los tratamientos 101-14M, 196-17C y Fercal han mostrado un valor significativamente superior, en torno a $23,8^{\circ}$ brix, que $161-49 \mathrm{C}, 41 \mathrm{~B}$ y RGM, en torno a $23,2^{\circ}$ brix.

El $\mathrm{pH}$ del mosto ha mostrado algunas diferencias estadísticamente significativas entre los portainjertos 196-17C, 41B y RGM, con valores medios cercanos a 3,60, y Fercal, con un valor medio de 3,44. La acidez titulable ha mostrado diferencias estadísticamente significativas entre los portainjertos Fercal, Gravesac y 161-49C, con valores superiores a 6,0 g/L, y los portainjertos RGM, 3309C, 101-14M, 41B y 196-17C, con valores por debajo de $5,6 \mathrm{~g} / \mathrm{L}$.

El ácido tartárico en mosto apenas mostró diferencias entre tratamientos, aunque se observó una tendencia 
Tabla 4. Sólidos solubles totales, SST ( ${ }^{\circ}$ brix); pH; Acidez titulable, A.T. (g $\left.\mathrm{TH}_{2} / \mathrm{L}\right)$; Acido tartárico, $\mathrm{TH}_{2}(\mathrm{~g} / \mathrm{L})$; Acido málico, $\mathrm{MH}_{2}(\mathrm{~g} / \mathrm{L})$; Potasio, $\mathrm{K}(\mathrm{mg} / \mathrm{L})$; de los 10 portainjertos (PI). Promedio del periodo 2010-2014.

\begin{tabular}{ccccccc}
\hline PI & SST & pH & A.T. & TH $_{2}$ & $\mathbf{M H}_{2}$ & K \\
\hline 110R & $23,6^{\mathrm{ab}}$ & $3,50^{\mathrm{ab}}$ & $5,90^{\mathrm{ab}}$ & 8,39 & $1,73^{\mathrm{ab}}$ & $1778^{\mathrm{ab}}$ \\
$\mathbf{1 0 1 - 1 4 M}$ & $23,9^{\mathrm{a}}$ & $3,54^{\mathrm{ab}}$ & $5,56^{\mathrm{b}}$ & 8,27 & $1,69^{\mathrm{b}}$ & $1803^{\mathrm{ab}}$ \\
$\mathbf{4 2 0 A}$ & $23,4^{\mathrm{ab}}$ & $3,49^{\mathrm{ab}}$ & $5,80^{\mathrm{ab}}$ & 8,35 & $1,78^{\mathrm{ab}}$ & $1759^{\mathrm{b}}$ \\
$\mathbf{3 3 0 9 C}$ & $23,5^{\mathrm{ab}}$ & $3,56^{\mathrm{a}}$ & $5,56^{\mathrm{b}}$ & 8,23 & $1,64^{\mathrm{b}}$ & $1760^{\mathrm{b}}$ \\
41B & $23,3^{\mathrm{b}}$ & $3,58^{\mathrm{a}}$ & $5,60^{\mathrm{b}}$ & 8,30 & $1,61^{\mathrm{b}}$ & $1713^{\mathrm{b}}$ \\
$\mathbf{1 6 1 - 4 9 C}$ & $23,2^{\mathrm{b}}$ & $3,52^{\mathrm{ab}}$ & $6,05^{\mathrm{a}}$ & 8,69 & $1,84^{\mathrm{ab}}$ & $1757^{\mathrm{b}}$ \\
$\mathbf{1 9 6 - 1 7 C}$ & $23,7^{\mathrm{a}}$ & $3,60^{\mathrm{a}}$ & $5,62^{\mathrm{b}}$ & 8,35 & $1,81^{\mathrm{ab}}$ & $1838^{\mathrm{a}}$ \\
Fercal & $23,7^{\mathrm{a}}$ & $3,44^{\mathrm{b}}$ & $6,19^{\mathrm{a}}$ & 8,70 & $1,99^{\mathrm{a}}$ & $1827^{\mathrm{a}}$ \\
Gravesac & $23,5^{\mathrm{ab}}$ & $3,49^{\mathrm{ab}}$ & $6,07^{\mathrm{a}}$ & 8,17 & $2,08^{\mathrm{a}}$ & $1829^{\mathrm{a}}$ \\
RGM & $23,3^{\mathrm{b}}$ & $3,57^{\mathrm{a}}$ & $5,52^{\mathrm{b}}$ & 8,22 & $1,67^{\mathrm{b}}$ & $1783^{\mathrm{ab}}$ \\
Sig & $*$ & $*$ & $*$ & - & $*$ & $*$ \\
\hline
\end{tabular}

a ser más elevado en Fercal y 161-49C, con valores medios en torno a $8,7 \mathrm{~g} / \mathrm{L}$, mientras que en el resto de portainjertos estuvo entre 8,2 y 8,4g/L. El ácido málico fue responsable en gran medida de la tendencia observada en la acidez total, ya que mostró diferencias estadísticamente significativas entre algunos tratamientos. Así, los portainjertos Gravesac y Fercal presentaron valores medios claramente más elevados, en torno a 2,04 g/L, que 41B, 3309C, RGM y 101-14M, con valores en torno a 1,65 g/L. El contenido en potasio del mosto mostró diferencias estadísticamente significativas favorables a los portainjertos 196-17C, Gravesac y Fercal, con valores en torno a $1830 \mathrm{mg} / \mathrm{L}$, con respecto a 41B, 161-49C, 420A y 3309C, con valores en torno a $1750 \mathrm{mg} / \mathrm{L}$.

\section{Conclusiones}

El empleo de distintos portainjertos de vid afectó al desarrollo vegetativo-productivo y a la calidad de la uva del cv. Sauvignon Blanc de forma variable. Los portainjertos 420A y 41B mostraron un potencial productivo medio más del 50\% superior que 196-17C y 161-49C, y más del 100\% superior que RGM. Las diferencias en el rendimiento fueron debidas al número de racimos por cepa $\mathrm{y}$, en mayor cuantía, al peso del racimo. Los portainjertos Fercal y Gravesac presentaron incremento medio del peso de madera de poda del $24 \%$ con respecto a $196-17 \mathrm{C}$ y 161-49C, y del 90\% con respecto a RGM. Las diferencias en la madera de poda fueron debidas mayoritariamente al vigor del sarmiento.

En cuanto a calidad de la uva, la concentración de azúcares se vio favorecida por 101-14M, 196-17C y Fercal y perjudicada por 161-49C, 41B y RGM. El pH del mosto fue favorecido por 196-17C, 41B y RGM y desfavorecida por Fercal. La acidez titulable fue incrementada por Fercal, Gravesac y 161-49C y disminuida por RGM, 3309C, 101-14M, 41B y 196-17C. El ácido tartárico apenas se vio afectado, aunque Fercal y 161-49C mostraron un valor medio superior. El ácido málico aumentó con Gravesac y Fercal y se redujo con 41B, 3309C, RGM y 101-14M. El contenido en Potasio se incrementó con 196-17C, Gravesac y Fercal y se vio reducido con 41B, 161-49C, 420A y 3309C.

En resumen, los efectos observados en los distintos parámetros analizados muestran la posibilidad de distintas alternativas de elección de portainjerto para el cultivo de Sauvignon Blanc en función de las condiciones medioambientales y los objetivos productivos del viñedo.

El desarrollo de este trabajo ha sido posible gracias a la colaboración del Grupo Yllera, propietario del viñedo, así como a la aportación analítica de la Estación Enológica de Castilla y León (Rueda, Valladolid), la contribución financiera de la Junta de Castilla y León y del FEDER, y por supuesto, la ayuda del personal del Grupo de Viticultura del ITACyL.

\section{Referencias}

[1] P. May. "Using grapevine rootstocks. The Australian perspective". Winetitles, Cowandilla SA (Australia), 62 p. (1994)

[2] P. Galet. "Grape varieties and rootstocks varieties". Oenoplurimédia, Chaintré (France), 315 p. (1998)

[3] A. Reynier. "Manual de viticultura". Ed. MundiPrensa, Madrid (España), 497 p. (2002)

[4] N. Dry. "Grapevine rootstocks. Selection and management for South Australian vineyards". Hyde Park Press, Adelaide (Australia), 85 p. (2007)

[5] T. Sampaio, C. Vasconcelos. "Optimizing water status, gas-exchange, fuit yield and composition using rootstocks". 14 $4^{\text {th }}$ GiESCO Symposium, Geisenheim (Germany), August 23-27 (2005)

[6] M.V. Alburquerque, F.J. Castaño, J. Yuste. "Influencia de diez portainjertos sobre el comportamiento de la variedad Tempranillo. Desarrollo productivo y vegetativo y calidad de la uva". Vida Rural 305, 52-56 (2010)

[7] J. Yuste, M.V. Alburquerque. "Influence of 9 grapevine rootstocks on vegetative-productive development and grape quality of Tempranillo variety in the A.O. Rueda (Spain)". Le progrès agricole et viticole: Special $17^{\text {th }}$ GiESCO Symposium, AstiAlba (Italy), August 29 - September 2, pp: 455-458 (2011)

[8] J. Yuste, M.V. Alburquerque. "Influencia de 10 portainjertos de vid sobre el desarrollo productivo y vegetativo y la calidad de la uva de cv. Tempranillo en la denominación de origen Toro (España)". Enoviticultura 22, 6-15 (2013)

[9] J. Yuste, M.V. Alburquerque. "Effects of 9 grapevine rootstocks on vegetative development, production and grape quality of cv. Tempranillo in the A.O. Cigales (Spain)". X Int. Terroir Congress, Budapest (Hungary), July 7-10 (2014)

[10] J. Yuste, E. Barajas, M.V. Alburquerque. "Influencia de 10 portainjertos de vid sobre el desarrollo productivo, la producción y la calidad de la uva del cv. Sauvignon blanc en la DO Rueda”. Enoviticultura 25, 7-13 (2013)

[11] J. Yuste, M.V. Alburquerque. "Rootstocks of grapevines: effects on vegetative development, production and grape quality of $\mathrm{cv}$. Verdejo in the D.O. Rueda (Spain)". 19 $9^{\text {th }}$ GiESCO Symposium, Gruissan (France), June 1-5 (2015)

[12] J. Yuste, R. Yuste, M.V. Alburquerque. "Ten grapevine rootstocks: effects on vegetative development, production and grape quality of $\mathrm{cv}$. Mencia in the D.O. Bierzo (Spain)". XI Int. Terroir Congress, McMinnville, OR (USA), July 10-14 (2016) 